\title{
Adapting to the COVID-19 Pandemic: Continuing HIV Prevention Services for Adolescents Through Telemonitoring, Brazil
}

\author{
Inês Dourado ${ }^{1} \cdot$ Laio Magno $^{1,2} \cdot$ Fabiane Soares $^{1} \cdot$ Paula Massa $^{3} \cdot$ Amy Nunn $^{4} \cdot$ Shona Dalal $^{5}$. \\ Alexandre Grangeiro ${ }^{3}$ on behalf of The Brazilian PrEP1519 Study Group
}

Published online: 21 May 2020

(c) Springer Science+Business Media, LLC, part of Springer Nature 2020

The COVID-19 pandemic is impacting the delivery of health care services everywhere, and although in the early stages, the results of disruptions are becoming clearer. Infectious disease and sexual health providers may be diverted to care for COVID-19 patients. Closing sexual health services due to COVID-19 quarantine policies will likely have detrimental impacts on health services access and discontinuity of care among marginalized populations in low- and middleincome countries [1]. These challenges may be particularly acute for sexual and gender minorities such as men who have sex with men (MSM) and transgender women (TGW), but also for sexual and reproductive health and family planning services for women [2-5]. Access to condoms as well as antiretroviral drugs and antibiotics to treat sexually transmitted infections (STIs) and HIV may also be affected, and may ultimately undermine HIV pre-exposure prophylaxis (PrEP) initiation and persistence $[3,6]$.

On March 20th, 2020 the Brazilian Ministry of Health $(\mathrm{MoH})$ announced that all Brazilian states had coronavirus cases [7], and issued guidelines on social distancing measures, such as voluntary home quarantine, and social isolation

The members of the The Brazilian PrEP1519 Study Group study group are listed in the acknowledgements.

Inês Dourado

ines.dourado@gmail.com

1 Instituto de Saúde Coletiva, Universidade Federal da Bahia, Salvador, Bahia, Brazil

2 Departamento de Ciências da Vida, Universidade do Estado da Bahia, Salvador, Bahia, Brazil

3 Faculdade de Medicina, Universidade de São Paulo, São Paulo, Brazil

4 School of Public Health, Brown University, Providence, RI, USA

5 Global HIV, Hepatitis and Sexually Transmitted Infections Programmes, World Health Organization, Geneva, Switzerland
[8]. On May 20th, the country had accumulated 271,628 confirmed cases of COVID-19 (129.3 cases/100.000 inhabitants) and 17,971 COVID-19 related deaths, fatality rate of $6.6 \%$ [9]. Brazil has one of the highest transmission rates of the virus in the world [10], but faces an uncoordinated national government response [11, 12]. On the other hand, the existence of a National Health System (SUS) [13] favors state and city governments to adopt their own approaches that range from voluntary social isolation to partial lockdown.

PrEP1519 is the first demonstration cohort study in Latin America exploring acceptability, use and PrEP persistence amongst adolescents MSM and TGW age 15-19 years oldadolescent key populations (AKP). While Brazil launched PrEP in its national health system in 2018, PrEP is not yet available for adolescents under age 18 [14]. PrEP1519 is ongoing in three cities in Brazil: Salvador (SSA), São Paulo (SP), and Belo Horizonte. Data collection started in March 2019 (SP); in April 2020 (SSA), 1949 were reached by recruitment strategies; 484 (24.8\%) participants have been enrolled: 383 in PrEP and 101 in non-PrEP arm. Of these, 91.5\% self-identified as MSM, $8.5 \%$ as TGW, $12.7 \%$ were between 15 and 17 years old and $87.3 \%$ 18-19 years old; $81.7 \%$ reported condomless anal sex at baseline.

With launch of this new PrEP demonstration program, customized PrEP clinics began new services and outreach programs tailored to AKP. Participants are recruited by several strategies, including: engaging peer-educators at schools and venues where young people congregate; social media platforms such as Instagram, Facebook, WhatsApp, and hook up apps; word-of-mouth, and by an artificial intelligence chatbot named "Amanda Selfie" conceived as a transgender woman. It is the first Latin America transgender chatbot. Available 24/7 on Facebook Messenger, it emulates chatbased conversations on sensitive subjects: sex, STIs, PrEP and combination prevention. It can identify those in higher risk for HIV, and schedule 
appointments to PrEP1519 clinics. Participants self-select into two arms: a PrEP arm and a non-PrEP arm. Participants in the non-PrEP arm are PrEP-eligible but chose not to use prophylaxis and receive other HIV combination prevention (counseling, condoms, lubricant, douche, and HIV self-test-HIVST). After PrEP initiation, participants are assigned a study peer-navigator and are monitored by the health team (Table 1). These activities take place in person, via smartphone, and on WhatsApp, Instagram, and Facebook messages.

All cities where PrEP1519 takes place have adopted quarantine and social isolation guidelines, including reductions in public transportation and suspension of many non-essential health services. Therefore, the difficulty for individuals, and marginalized groups in particular, to reach services is much harder now. The PrEP1519 study decided to continue its work during to the pandemic, and was able to adapt quickly to the new situation due to the social media and telemonitoring infrastructure that was set up to reach AKP beforehand. In this paper, we aim to describe the strategies adopted by PrEP1519- sites of Salvador and São Paulo to continue to provide HIV and sexual and reproductive health services during quarantine periods to contain the COVID19 pandemic.

\section{Contingency Measures Adapted for COVID-19}

The contingency plan was adapted from revised protocols from different countries that showed effective telehealth interventions [15-18]. In order to minimize as much as possible the effect of quarantine measures on PrEP initiation, on PrEP continuation, and access to other sexual health care and prevention needs, we immediately amended our research procedures and care services. Salvador and São Paulo now provides additional telehealth as the default care provision platforms, such as digital participant recruitment and peer support and navigation via smartphone text messages and online social media (Instagram and Facebook), streamlined PrEP initiation and retention procedures. The strategies of navigation online were more intensified as adolescents are eager to have a conversation and ask questions on how to persist with their PrEP use under quarantine. Providers still offer in person visits at their discretion, and reserve care for individuals who do not have respiratory symptoms (Table 1).

PrEP1519 collects all data digitally; this data is fully integrated with electronic medical record platforms (www.sispr ep1519.org). Even though, the team can monitor the participant's follow-up and can have access to clinical data in real time, it has been essential at this moment for the work from home (home-office).

\section{PrEP1519 Telehealth Strategy: Opportunity to Maintain HIV/STI Prevention and PrEP Delivery Services}

Figure 1 highlights our strategy. We now use e-services and home delivery of condoms, lubricant, douche, HIVST and PrEP medications. HIVST is available for AKP contacted by social media interactions, and for PrEP users. A package with HIVST and guidance is dispensed [19] for those who signed an online consent form, by fast delivery services or mail depending on the participants' preferred address, and has no mention of the content. For those under 18 years old, we ask for parental informed consent whenever necessary for the follow-up of the adolescent in the study. Peer-educators and peer-navigators are responsible for online recruitment, and linkage to the PrEP clinic. Peer-navigators and PrEP1519 health team (doctor, nurse, psychologist, pharmacist, and social worker) offer online support for participants.

\section{Understanding HIV/STI Risk and Creating Demand Virtually in Times of COVID-19 Pandemic}

The strategies of recruitment during the COVID-19 pandemic are described in Table 1. These interactions aim to identify adolescents searching for socialization, for sexual encounters, and those engaged in private sex parties for sex during quarantine [20]. The monitoring of online social media platforms and hook up apps has shown that, although there is a reduction, the number of AKP who use these platforms during quarantine to interact socially or look for sexual partners is significant.

\section{Clinical Follow-up PrEP Initiation and PrEP Continuation}

For PrEP initiation, a face to face appointment is scheduled, and follows the routine pre COVID-19 with adjustments described in Table 1. These adaptations allow AKP who choose to initiate PrEP to reduce in person appointment time at the clinic by approximately $50 \%$.

For PrEP continuation, those who the health team identify as eligible (participant with high PrEP adherence, no suspicion of acute HIV infection and no severe side effects), a telehealth appointment is scheduled, and PrEP medication and HIVST delivered to their preferred addresses. Those who are not eligible, or participant does 
Table 1 Provision of services at PrEP1519 clinics before and after COVID-19

Original before the pandemic

Recruitment

Recruitment based on the activities of peer-educators: with youth at venues and schools; the use of social media platforms (Instagram, Facebook, WhatsApp, YouTube, Tik Tok, Spotify) and hook up apps (Grindr, Tinder, Hornet, and Badoo)

Recruitment based on artificial intelligence-Amanda Selfie chatbotthrough sponsored posts in Instagram promoting key prevention messages (HIV and COVID-19) with focus on AKP

Community mobilization by demand creation of PrEP at LGBTQ parties and venues, promotional videos and flyers on social media platforms (Instagram and YouTube) and radio programs on Spotify

After an AKP is identified, PrEP and other sexual health services are offered and based on the choice, an appointment was scheduled at PrEP1519 clinics

\section{Navigation}

After PrEP initiation, a peer-navigator is assigned to follow-up the needs of each participant. The follow-up is mostly online, through social media platforms and smartphone text messages. They emphasizes and monitors PrEP adherence at PrEP initiation, and PrEP continuation biweekly and monthly. The nurse and the doctor supervise this work. The communication channels between participants and peer-navigators are open to allow participants to initiate a conversation according to their needs

\section{Provision of services}

PrEP clinic-operational hours

Salvador site: Monday to Friday from 9 am to 5 pm; São Paulo site: Monday to Friday from 10 am to $7 \mathrm{pm}$

No worries with social distance

Health care providers (doctor, nurse, psychologist, pharmacist, social worker, lab technician) working every day of the week

Public transportation reimbursement

PrEP initiation

For PrEP enrolment, face to face appointments were scheduled by the participant, or walk-ins. Eligibility assessment for PrEP use, clinical evaluation, HIV rapid tests, collect a blood sample to STI serological tests such as HIV, syphilis, viral hepatitis and HTLV, pharyngeal, rectal and urethral swabs for chlamydia and gonorrhea tests, and safety tests for PrEP use that assess kidney and liver function, and dispensation of PrEP for 1 month
COVID-19 change (26th February 2020)

Recruitment based on the activities of peer-educators in social media platforms and hook up apps only

Maintained

Promotional videos on social media platforms, radio programs on Spotify and, "Lives" and IGTV in the Instagram, led by peer-educators featuring as drag queens and digital influences. They use an LGBTQ culture approach and talk about sex, hormone therapy, and art focused on AKP. Moreover, messages, photos, podcasts, playlists, and flyers posted in Project Instagram has had great success to reach new and young LGBTQ followers (https://www.instagram.com/preparasal vador/?hl=ptbr and https://instagram.com/vcprepsp?igshid=dyf76 oj8lef7)

After an AKP is identified, PrEP and other sexual health services are offered

If they are interested in the provision of services, a telehealth appointment is scheduled to conduct an eligibility assessment for PrEP use; and a face to face appointment is scheduled at PrEP1519 clinics

If they are not interested in the provision of services during the quarantine, the contact information is collected in order to create a list of potential PrEP users to be contacted after the quarantine

And we offer a prevention kit with an HIVST, condoms, lubricants, PrEP flyer, anal douche and hand sanitizer to be delivered to their preferred address after online signed informed consent

Maintained. But the follow-up is only online through social media platforms and smartphone text messages

Peer-navigators helps to schedule face-to-face appointments and/or telehealth

Peer-navigators inquires: on COVID-19 symptoms; close physical contact with someone diagnosed with COVID-19; cohabitating with family members or with someone vulnerable to COVID-19

\section{PrEP clinic-operational hours}

Salvador site: two days of the week from 9 am to 2 pm for scheduled face to face appointments only. São Paulo site: Monday to Friday from $10 \mathrm{am}$ to $7 \mathrm{pm}$-open door was maintained, but most in person appointments are scheduled

The organization of the reception chairs respects the distance of at least two and a half meters, and all spaces have hand sanitizer. Site staff advises participants on safety measures to be observed on public transportation

Rotation days for health care providers

Choice of public or private transportation reimbursement

For PrEP enrolment, a telehealth appointment is scheduled to conduct an eligibility assessment for PrEP use. Then face to face appointments are scheduled to conduct HIV rapid tests, collect a blood sample to STI serological tests such as HIV, syphilis, viral hepatitis and HTLV, and safety tests for PrEP use that assess kidney and liver function, and dispensation of PrEP for 1 or 2 months 
Table 1 (continued)

Original before the pandemic

No worries with temperature measurements of participants on arrival

Face to face interviews for socio-behavioral questionnaires at PrEP clinics only

No worries with the COVID-19 symptoms

No worries with temperature measurements of participants on arrival PrEP continuation

Participants attended follow up visits after baseline (1st month and then every 3 months) without previous online evaluation. At each visit: clinical evaluation, HIV rapid tests, collect blood samples to STI serological tests such as HIV, syphilis, viral hepatitis and HTLV and for safety tests for PrEP use that assess kidney and liver function; collect DBS for future PK substudies; pharyngeal, rectal and urethral swabs for chlamydia and gonorrhea tests; and dispensation of PrEP for 3 month at PrEP clinics only

Interviews for socio-behavioral questionnaires at PrEP clinics only

No worries with the COVID-19 symptoms

No worries with temperature measurements of participants on arrival
COVID-19 change (26th February 2020)

The temperature of all participants is measured on arrival

Socio-behavioral questionnaires to be completed online; monitored by team members

Site staff conducts a phone call to all scheduled participants in the day before their appointment to confirm their intention to attend the visit, and evaluates if s/he lives with someone at a higher risk of COVID19 (e.g. the elderly or people with comorbidities) and offers guidance on COVID-19 protection for in person appointments. Also to inquire about COVID-19 symptoms: fever $\left(>37.7^{\circ} \mathrm{C}\right)$, cough, runny nose, shortness of breath or difficulty breathing, muscle pain, or anosmia. If any of these symptoms are reported, his/her visit is postponed and s/ he receives guidance on COVID-19 management and resources

The temperature of all participants is measured on arrival

All PrEP users are contacted to inquire about their interest in telehealth and delivery of PrEP pills and HIVST or to confirm a face-to-face appointment. For participants who chose telehealth to continue their use of PrEP, they are evaluated by the health team for any clinical impediments. If none, two HIVST and 120 PrEP pills are mailed to the preferred address on online forms that also contain an informed consent to be checked. Health team reinforce that it is necessary to perform the HIVST, to send the negative HIV test result to the doctor before opening a new PrEP bottle, and to repeat this procedure quarterly until quarantine measures are over

Socio-behavioral questionnaires to be completed online; monitored by team members

If participant's choice is a face-to-face appointment, we conduct HIV rapid tests, collect a blood sample to STI serological tests such as HIV, syphilis, viral hepatitis and HTLV, and safety tests for PrEP use that assess kidney and liver function, collect DBS for future for future PK sub-studies; and dispensation of PrEP for 1 or 2 months. Site staff conducts a phone call the day before their appointment to confirm their intention to attend the visit, and evaluates if $\mathrm{s} / \mathrm{he}$ lives with someone at a higher risk of COVID-19 (e.g. the elderly or people with comorbidities) and offers guidance on COVID-19 protection for in person appointments. Also to inquire about COVID-19 symptoms: fever $\left(>37.7^{\circ} \mathrm{C}\right)$, cough, runny nose, shortness of breath or difficulty breathing, muscle pain, or anosmia. If any of these symptoms are reported, his/her visit is postponed and s/he receives guidance on COVID-19 management and resources, and the clinical team evaluates the possibility of sending PrEP and HIVST to their preferred addresses

The temperature of all participants is measured on arrival not want telehealth, a face to face appointment is scheduled, and follows the same routine as for PrEP initiators. In cases of suspecting COVID-19 infection, we follow the telehealth routine for eligible participants (Table 1).

Telehealth appointments are conducted according to the adolescent's availability and based on health providers' evaluation, through a video or voice connection, including general health screening, PrEP and other strategies of HIV prevention such as counseling, HIVST and COVID-19 prevention guidance. Telehealth is the main intervention for PrEP follow-up visits to eligible participants but exceptionally in person for new PrEP initiators (30 days follow up).

\section{Adding of Social and Mental Health Support into Telehealth}

Because social distancing can exacerbate or precipitate the symptoms of depression and anxiety [21] and participants may also be at risk for domestic violence because of potential disclosure of their gender identity, social and mental health support service is now available via telehealth, and conducted by a member of the health team depending on the severity of the situation. So far, participants in need of support, have switched to the e-service modality without 


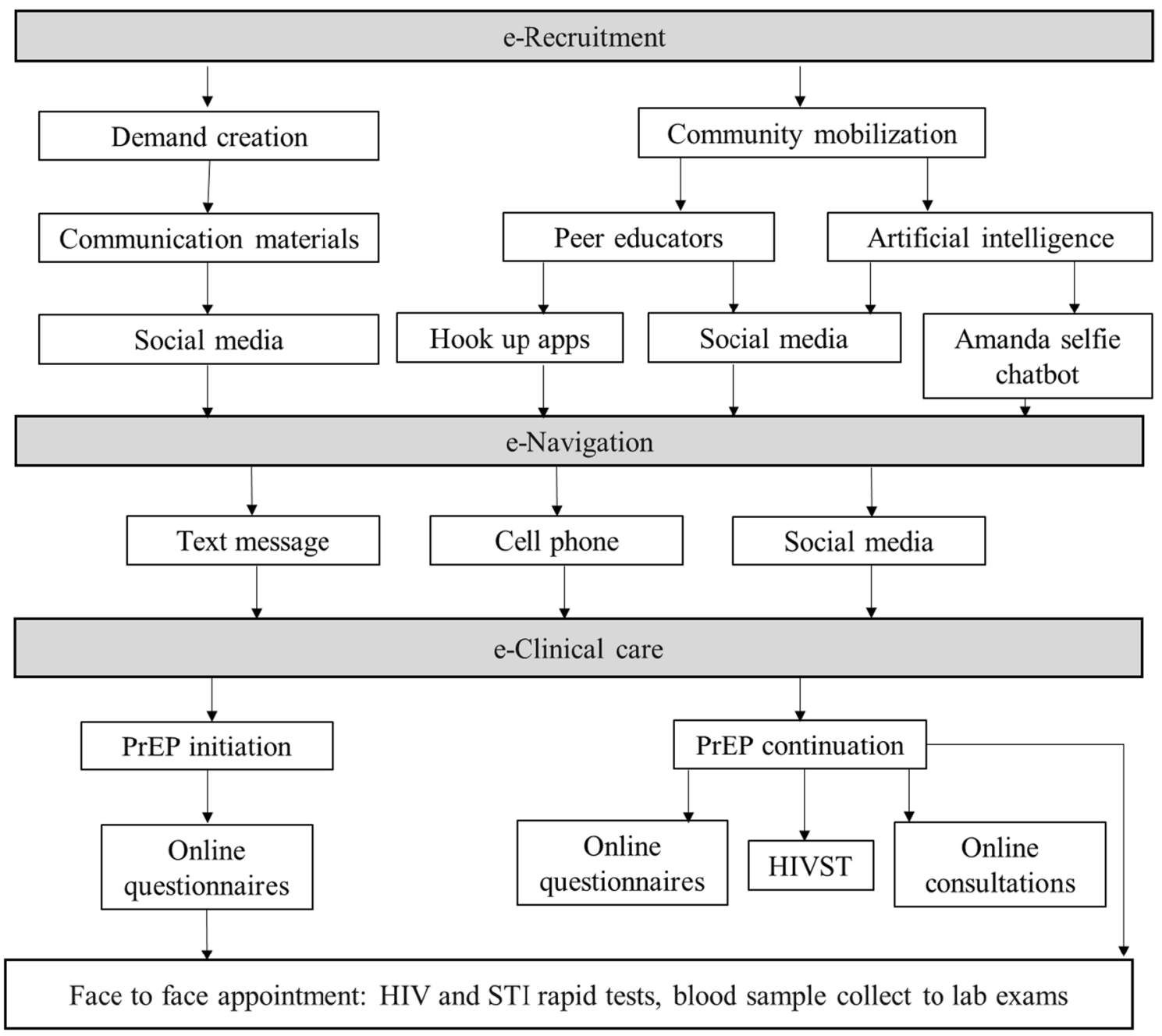

Fig. 1 The PrEP1519 telehealth strategy

constraints. Furthermore, mental health support short videos developed by communication team are offered.

\section{Lessons Learned}

Because of the investments already made in demand creation, and online social media platforms for marginalized adolescents by the PrEP1519 project, the rapid shift to more complete telehealth services due to COVID-19 was possible. This adaptation has allowed PrEP initiation and PrEP continuation for AKP. Nevertheless, some reduction in recruitment of new participants for the study is observed. An added benefit is that project staff have been able to be retained and continue to provide services.

Keen demand for PrEP and other HIV prevention services continues among AKP in Brazil. The adoption of wraparound telehealth platforms has simplified and streamlined procedures for PrEP initiation, and has facilitated and enabled continuity in PrEP services during quarantine. These changes have been well-received by our participants and providers and will likely be sustained even after quarantine restrictions are lifted. These experiences offer important lessons for other middle income countries, as well as other countries scaling up PrEP.

Acknowledgements We are grateful to the adolescents who participate in this study and their parents and guardians, to UNITAID that funds the PrEP1519 study, to the Brazilian National Health System (SUS) that donate PrEP medications, condoms, rapid tests and provide additional care services for PrEP1519 participants, through the Department of Chronic Diseases and Sexually Transmitted Infections of the Ministry of Health, the Bahia State and Salvador City Department of Health, the São Paulo State and City Department of Health, and the city of São Paulo AIDS Program. To Fiocruz and FIOTEC that supports the study.

*The Brazilian PrEP1519 Study Group: Salvador site: Luís Augusto V. da Silva, Priscilla Caires, Carlos Jefferson, Filipe Mateus Duarte, Pedro Rafael Nascimento, Thaís Fonseca, Fabiane Neves Fernandes, Lucília Nascimento. São Paulo site: Regis Alves da Silveira, Victor Guilherme Lopes da Silva Lomba, Raphaella Goulart, Eduardo Araújo Oliveira, Audre Verneck, Luiz Felipe Alves de Sousa, André Araújo da 
Silva, Solange de Souza Queiroz Belo Horizonte site: Dirceu Greco, Unaí Tupinambás, Mateus Westin, Marília Greco, Yuppiel Martinez

\section{References}

1. Hall KS, Samari G, Garbers S, Casey SE, Dixon Diallo D, Orcutt $\mathrm{M}$, et al. Centring sexual and reproductive health and justice in the global COVID-19 response. Lancet. 2020. https://doi.org/10.1016/ S0140-6736(20)30801-1.

2. Sanchez TH, Zlotorzynska M, Rai M, Baral SD. Characterizing the Impact of COVID-19 on men who have sex with men across the United States in April, 2020. AIDS Behav. 2020. https://doi. org/10.1007/s10461-020-02894-2.

3. Hussein J. COVID-19: What implications for sexual and reproductive health and rights globally? Sex Reprod Health Matters. 2020;28:3.

4. Tang K, Gaoshan J, Ahonsi B. Sexual and reproductive health (SRH): a key issue in the emergency response to the coronavirus disease (COVID-19) outbreak. Reprod Health. 2020;17:1-3.

5. Perez-Brumer A, Silva-Santisteban A. COVID-19 policies can perpetuate violence against transgender communities: insights from Peru. AIDS Behav. 2020;1:3.

6. Ahmed Z, Sonfield A. The COVID-19 outbreak: potential fallout for sexual and reproductive health and rights. Guttmacher: Guttmacher Institute; 2020.

7. BRASIL. Portaria No 454, de 20 de Março de 2020. Brasília: Ministério da Saúde. 2020a.

8. BRASIL. Portaria No 356, de 11 de Março de 2020. Brasília: Ministério da Saúde. 2020c.

9. Painel de casos de doença pelo coronavírus 2019 (COVID-19) no Brasil. Ministério da Saúde. 2020. https://covid.saude.gov.br/. Accessed 20 May 2020.

10. Rede CoVida. Ciência, Informação e Solidariedade. Painel Coronavírus. 2020. https://www.covid19br.org. Accessed 11 May 2020.

11. The Lancet. COVID-19 in Brazil: "So what?". Lancet. 2020;395(10235):1461.

12. Werneck GL, Marília SC. A pandemia de COVID-19 no Brasil: crônica de uma crise sanitária anunciada. Cad Saúde Pública. 2020;36(5):e00068820.
13. Castro MC, Massuda A, Almeida G, Menezes-Filho NA, Andrade MV, de Souza Noronha KVM, et al. Brazil's unified health system: the first 30 years and prospects for the future. Lancet. 2019;394(10195):345-56.

14. BRASIL. Clinical Protocol and Therapeutic Guidelines for PreExposure Prophylaxis (PrEP) for Risk of HIV Infection. Secretaria de Vigilância em Saúde Departamento de IST e HIV: Ministério da Saúde. 2017.

15. Dandachi D, Freytag J, Giordano TP, Dang BN. It is time to include Telehealth in our measure of patient retention in HIV CARE. AIDS Behav. 2020. https://doi.org/10.1007/s10461-02002880-8.

16. Kay ES, Musgrove K. From HIV to coronavirus: AIDS service organizations adaptative responses to COVID-19, Birmingham, Alabama. AIDS Behav. 2020;1:3.

17. Peavy KM, Darnton J, Grekin P, Russo M, Green CJB, Merrill $\mathrm{JO}$, et al. Rapid to implementation of service delivery changes mitigate COVID-19 and maintain access to methadone among persons with and at high-risk for HIV in an opioid treatment program. AIDS Behav. 2020;1:4.

18. Rogers BG, Coats CS, Adams E, Murphy M, Stewart C, Arnold T, et al. Development of telemedicine infrastructure at an LGBTQ+ clinic to support HIV prevention and care in response to COVID19, Providence, RI. AIDS Behav. 2020;1:3.

19. PrEP1519. https://www.youtube.com/watch? $=\mathrm{iYCuQ09Cu6}$ $w \& t=2 s .2019$.

20. https://www.antropologicas-epidemicas.com.br/post/pandemiasexualidade-epercep\%C3\%A7\%C3\%A3o-do-risco-algumas-notas -sobre-quarentena-e-desejode. Pandemia, sexualidade e percepção do risco: algumas notas sobre quarentena e desejo-. 2020.

21. Venkatesh A, Edirappuli S. Social distancing in covid-19: what are the mental health implications? BMJ. 2020;369:m1379.

Publisher's Note Springer Nature remains neutral with regard to jurisdictional claims in published maps and institutional affiliations. 